\title{
Effectiveness of smoking cessation therapies: a systematic review and meta-analysis Ping $\mathrm{Wu}^{1}$, Kumanan Wilson ${ }^{2}$, Popey Dimoulas ${ }^{1}$ and Edward J Mills*1,3
}

Address: ${ }^{1}$ Department of Clinical Epidemiology and Biostatistics, McMaster University, Hamilton, Canada, ${ }^{2}$ Department of Medicine, University of Toronto, Toronto, Canada and ${ }^{3}$ Faculty of Health Sciences, Clinical Epidemiology \& Biostatistics, HSC-2C12, 1200 Main Street West, Hamilton, ON L8N 3Z5, Canada

Email: Ping Wu - wuping629@hotmail.com; Kumanan Wilson - Kumanan.Wilson@uhn.on.ca; Popey Dimoulas - popydg@gmail.com; Edward J Mills* - millsej@mcmaster.ca

* Corresponding author

Published: II December 2006

BMC Public Health 2006, 6:300 doi:10.I I86/I47|-2458-6-300
Received: II August 2006

Accepted: II December 2006

This article is available from: http://www.biomedcentral.com/I47I-2458/6/300

(c) 2006 Wu et al; licensee BioMed Central Ltd.

This is an Open Access article distributed under the terms of the Creative Commons Attribution License (http://creativecommons.org/licenses/by/2.0), which permits unrestricted use, distribution, and reproduction in any medium, provided the original work is properly cited.

\begin{abstract}
Background: Smoking remains the leading preventable cause of premature deaths. Several pharmacological interventions now exist to aid smokers in cessation. These include Nicotine Replacement Therapy [NRT], bupropion, and varenicline. We aimed to assess their relative efficacy in smoking cessation by conducting a systematic review and meta-analysis.
\end{abstract}

Methods: We searched 10 electronic medical databases (inception to Sept. 2006) and bibliographies of published reviews. We selected randomized controlled trials [RCTs] evaluating interventions for smoking cessation at I year, through chemical confirmation. Our primary endpoint was smoking cessation at I year. Secondary endpoints included short-term smoking cessation ( $\sim 3$ months) and adverse events. We conducted random-effects meta-analysis and metaregression. We compared treatment effects across interventions using head-to-head trials and when these did not exist, we calculated indirect comparisons.

Results: We identified 70 trials of NRT versus control at I year, Odds Ratio [OR] I.7I, 95\% Confidence Interval $[\mathrm{Cl}], \mathrm{I} .55-\mathrm{I} .88, \mathrm{P}=<0.000 \mathrm{I})$. This was consistent when examining all placebocontrolled trials (49 RCTs, OR I.78, 95\% Cl, I.60-I.99), NRT gum (OR I.60, 95\% Cl, I.37-I.86) or patch (OR I.63, 95\% Cl, I.4I-I.89). NRT also reduced smoking at 3 months (OR I.98, 95\% Cl, I.77-2.2I). Bupropion trials were superior to controls at I year (I2 RCTs, OR I.56, 95\% Cl, I.IO$2.2 \mathrm{I}, \mathrm{P}=0.0 \mathrm{I}$ ) and at 3 months (OR 2.13,95\% Cl, I.72-2.64). Two RCTs evaluated the superiority of bupropion versus NRT at I year (OR I.I4, 95\% Cl, 0.20-6.42).

Varenicline was superior to placebo at I year (4 RCTs, OR 2.96, 95\% Cl, 2.I2-4.12, P =< 0.000I) and also at approximately 3 months (OR $3.75,95 \% \mathrm{Cl}, 2.65-5.30)$. Three RCTs evaluated the effectiveness of varenicline versus bupropion at I year (OR $1.58,95 \% \mathrm{Cl}, 1.22-2.05)$ and at approximately 3 months (OR I.6I, 95\% Cl, I.I6-2.2I). Using indirect comparisons, varenicline was superior to NRT when compared to placebo controls (OR I.66, 95\% Cl I.17-2.36, P = 0.004) or to all controls at I year (OR I.73, 95\% Cl I.22-2.45, P = 0.00I). This was also the case for 3-month data. Adverse events were not systematically different across studies.

Conclusion: NRT, bupropion and varenicline all provide therapeutic effects in assisting with smoking cessation. Direct and indirect comparisons identify a hierarchy of effectiveness. 


\section{Background}

Smoking is the leading cause of preventable death in the world[1]. Given the multitude of health benefits of smoking cessation, considerable effort has been focused on identifying mechanisms to assist smokers in quitting. However, smoking cessation is challenging and behavioural interventions have had only modest success [2].

Drug therapy has been increasingly relied upon to assist in smoking cessation. The most common of these has been nicotine replacement therapy [NRT] [3]. More recently, attention has focused on the use of anti-depressant therapy and specifically the agent bupropion[4]. Five new trials have demonstrated the effectiveness of a new agent with a novel mechanism of action, varenicline, in improving cessation rates [5-9].

How effective are pharmacologic smoking cessation therapies? No systematic review and meta-analysis has examined this issue since the availability of information of the newest agent. We conducted a meta-analysis of Randomized Controlled Trials [RCTs] to identify the effectiveness of the various pharmacological interventions in improving cessation rates.

\section{Methods \\ Eligibility criteria}

Our primary outcome of interest was smoking cessation at 1 year. Our secondary outcomes were short-term smoking cessation defined as 3 months after initiating treatment or closest available data to that time point, within one month. Our additional secondary outcome evaluated adverse events. We included any RCT of NRT of any delivery method, bupropion or varenicline. We included only RCTs of at least 1 years duration with chemical confirmation of smoking cessation, as there is reason to doubt patient self-report regarding addictions. Methods of assessing smoking cessation also vary from study to study. The most common method is self-report, however, this can have false cessation rates as high as $30 \%[10]$. False reporting is most likely to occur in a trial setting or in assessing smoking status after a medical event. In both situations the smoker is under considerable pressure to quit. Laboratory tests are, therefore, often used to verify smoking status, especially in clinical trials. Methods of biological verification include serum and saliva thiocyanate (SCN), expired carbon monoxide (CO), plasma, saliva and urinary cotinine and plasma and urinary nicotine. Each of these have various strengths and weaknesses[11]. Studies had to report smoking cessation as either sustained abstinence at the time periods or point-prevalence of abstinence. When both outcomes were available, we considered sustained abstinence to be a superior clinical marker of abstinence. We excluded non-RCTs, post-hoc analyses, maintenance therapy, and studies that reported outcomes as self-report.

\section{Search strategy}

In consultation with a medical librarian (PR), we established a search strategy. We searched independently, in duplicate, the following 10 databases (from inception to September 10, 2006): MEDLINE, EMBASE, Cochrane CENTRAL, AMED, CINAHL, TOXNET, Development and Reproductive Toxicology, Hazardous Substances Databank, Psych-info and Web of Science, databases that included the full text of journals (OVID, ScienceDirect, and Ingenta, including articles in full text from approximately 1700 journals since 1993). In addition, we searched the bibliographies of published systematic reviews[3,12$18,4,19]$ and health technology assessments[20]. Searches were not limited by language, sex or age.

\section{Study selection}

Two investigators (EM, PW) working independently, in duplicate, scanned all abstracts and obtained the full text reports of records, that indicated or suggested that the study was a RCT evaluating a smoking cessation therapy on the outcomes of interest. After obtaining full reports of the candidate trials (either in full peer-reviewed publication or press article) the same reviewers independently assessed eligibility from full text papers.

\section{Data collection}

Two reviewers (PW, PD) conducted data extraction independently using a standardized pre-piloted form. Reviewers collected information about the smoking cessation intervention tested, the population studied (age, sex, underlying conditions), treatment dosages and dosing schedules, the treatment effect at 1 year and at 3 months, the specific measurement of abstinence (sustained or point-prevalence), and the chemical confirmation methods. Study evaluation included general methodological reporting quality features including allocation concealment, sequence generation, blinding status, intention-totreat, and appropriate descriptions of loss to follow-up. Quality of reporting could be considered as analogous to methodological quality if one assumes that failure to report on a component of study design (for example blinding) actually indicated that the component was not employed. We entered the data into an electronic database such that duplicate entries existed for each study; when the two entries did not match, we resolved differences through discussion and consensus.

\section{Data analysis}

In order to assess inter-rater reliability on inclusion of articles, we calculated the Phi statistic $(\varphi)$, which provides a measure of inter-observer agreement independent of chance[21]. We calculated the Odds Ratios [OR] and 
appropriate 95\% Confidence Intervals [CIs] of outcomes according to the number of events of abstinence reported in the original studies or sub-studies. In circumstances of zero outcome events in one arm of a trial, we added 1 to each arm, as suggested by Sheehe[22]. We first pooled studies of all NRT interventions versus all controls using the DerSimonian-Laird random effects method,[23] which recognizes and anchors studies as a sample of all potential studies, and incorporates an additional between-study component to the estimate of variability[24]. We calculated the $\mathrm{I}^{2}$ statistic for each analysis as a measure of the proportion of the overall variation that is attributable to between-study heterogeneity[25]. Forest plots are displayed for each primary analysis, showing individual study effect measures with 95\% CIs, and the overall DerSimmonian-Laird pooled estimate. We then conducted a meta-regression analysis on the NRT studies with predictors of heterogeneity including the following covariates: placebo control; reporting of sequence generation; reporting of allocation concealment; use of gum or patch; and, method of chemical confirmation of abstinence. When the meta-regression indicated heterogeneity, we conducted alternative sensitivity tests using z-tests to determine differences between the studies reporting the covariates to the pooled all-studies effect size. We additionally conducted separate pooled analyses of NRT versus placebo, gum versus control and patch versus control. We conducted all analyses at 1 year and also at 3 months. For bupropion trials, we pooled all bupropion trials (standard and sustained-release) versus all controls and conducted a meta-regression analysis using the following covariates: placebo control; reporting of sequence generation; reporting of allocation concealment; method of chemical confirmation of abstinence; and plans to quit. We conducted separate meta-regression analyses and calculated the relevant ORs for the covariates as the exponent of the point estimates[26]. The point estimates and 95\% $\mathrm{CI}$ around denote the expected change in the pooled effect size when the covariates are considered. The OR of the point estimates and 95\% CI confer the likelihood of the covariate affecting individual trial outcomes. We additionally pooled all placebo-controlled trials and evaluated effect sizes at 1 year and at 3 months. For head-to-head trials of bupropion versus NRT, we conducted pooled random-effects analyses at 1 year and at 3 months. For varenicline trials, we conducted pooled random-effects analyses of varenicline versus placebo at 1 year and at 3 months and for head-to-head trials of varenicline versus bupropion at 1 year and at 3 months. Head-to-head trials provide the strongest inferences regarding intervention superiority[27]. However, in the absence of head-to-head trials of varenicline versus NRT, we conducted indirect comparisons of these interventions versus placebo using methods described by Bucher et al and conducted z-tests to confirm[28]. This method maintains the randomiza- tion from each trial and compares the summary estimates of pooled interventions with CIs. We calculated adverse events, where reported, using Peto's Odds Ratio [OR] with 95\% CIs[29]. Analyses were conducted using StatsDirect (version 2.5.2) and Comprehensive Meta-analysis (version 2).

\section{Results}

We found 70 RCTs examining NRT versus control interventions (See Figure 1 and Additional File 1), [30-99] 49 of which compared NRT to placebo $[30,32,34,35,38,39,43,44,46,48,50,54,56-59,62,64-69$, 71-82,84-87,89-91,93,94,96,98,99] thirty one studies compared NRT to other controlled groups (See table 1) $[31,33,36,37,40-42,45,49,51-53,55,61,63,70,83,88,92$, $95,97]$ and 1 study uses both placebo and no intervention as control group[47].

Thirty-three studies evaluated NRT gum [30-49,51$53,55,61,63,64,71,83,87,95,96,98,99], 23$ evaluated NRT patch $[50,56,57,60,62,65,67-70,72,76,78-80,82,84,86$, $88,90,92,94,97]$. The remaining studies evaluated the efficacy of nicotine inhalers, nasal spray or lozenges $[54,58,59,66,73-75,77,81,85,89,91,93]$.

All of the studies provided sufficient details to evaluate NRT versus control at 1 year [30-99]. Fifty-nine provided sufficient details to evaluate NRT versus control at or about 3 months[30-33,36,37,39-41,44,47,49-54,56-59, 43,59-61,63-86,88-96,98,99].

We also found 11 studies evaluating bupropion versus placebo $[5,6,8,82,100-106]$ and one RCT evaluating bupropion with no intervention[97] (See Figure 1 and Additional File 2). Further, 2 of these evaluated bupropion versus NRT $[82,97]$.

Finally, we identified 4 studies evaluating varenicline versus placebo (See Figure 1 and Additional File 3) [5,6,8,9]. Of these, 3 also evaluated varenicline versus bupropion[5,6,8]. Agreement on original inclusion of all trials was excellent $(\varphi=0.88)$.

\section{Quality of methodological reporting}

Of the 70 trials assessing NRT versus controls, studies were varied in reporting important methodological features, including: sequence generation $(22 / 70)$, allocation concealment $(11 / 70)$, blinding status $(64 / 70)$, appropriate blinding (45/70), intention-to-treat $(67 / 70)$, and appropriate descriptions of loss to follow up $(44 / 70)$.

Studies assessing bupropion versus controls were similarly varied in reporting, including: sequence generation (4/12), allocation concealment (4/12), blinding status 
NRT

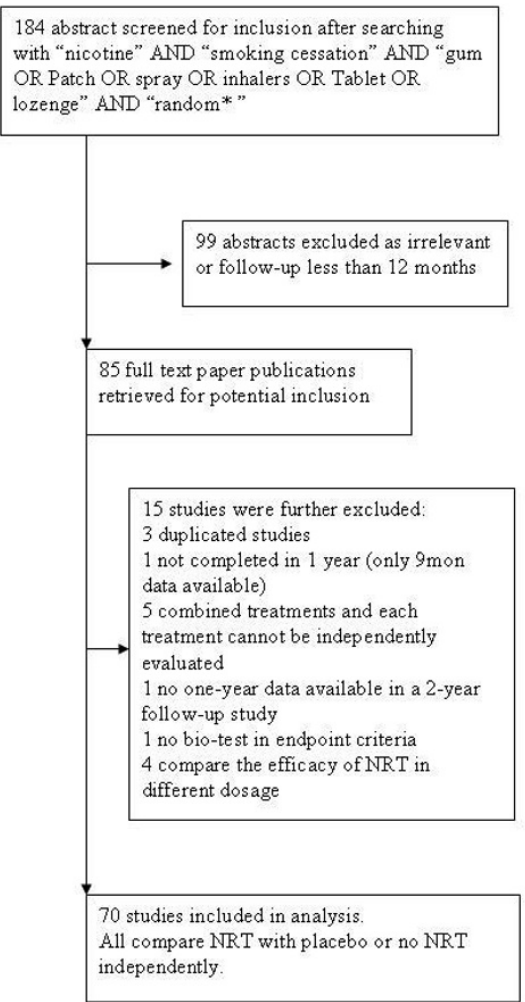

Bupropion

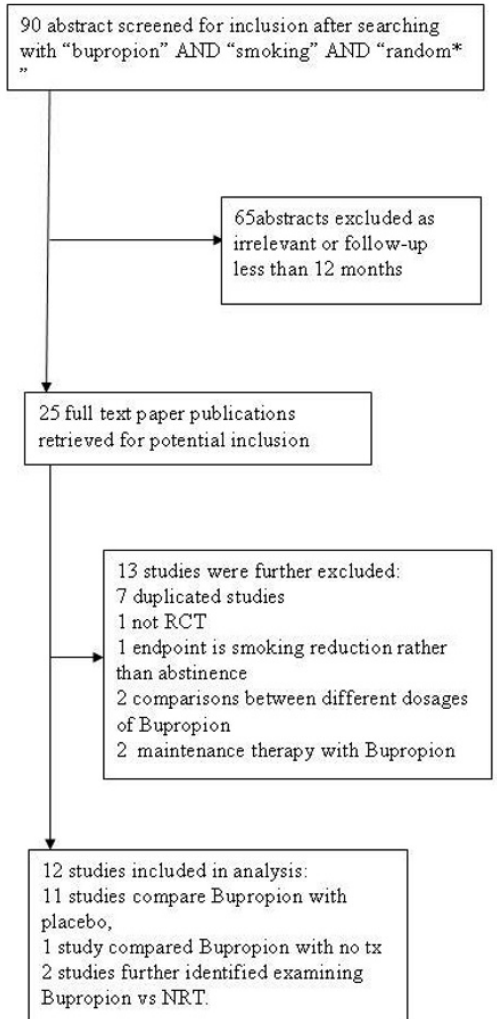

Varenicline

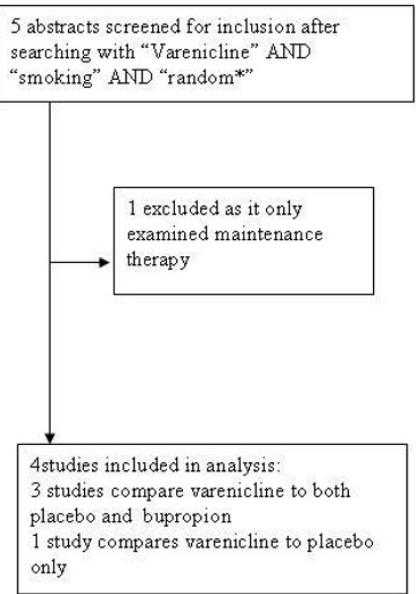

Figure I

Flowcharts of study searches.

$(11 / 14)$, intention to treat $(12 / 12)$ and appropriate descriptions of loss to follow up (10/12).

The 4 studies assessing varenicline appropriately reported the methodological criteria, except 1 study that did not report the method of sequence generation[9].

\section{Meta-analysis}

NRT

We combined 70 trials (total $\mathrm{n}=28,343$ ) assessing NRT versus controls at 1 year. The pooled OR of smoking cessation favored NRT over controls (OR 1.71,95\% CI, 1.55$1.88, \mathrm{P}=<0.0001, \mathrm{I}^{2}=26.5 \%$, Heterogeneity $\mathrm{P}=0.02$, See Figure 2). This was consistent when evaluating only placebo controlled NRT trials ( 49 trials, $\mathrm{n}=21,512$, OR 1.78, 95\% CI, 1.60-1.99, $\mathrm{P}=<0.0001, \mathrm{I}^{2} 27.4 \%$, Heterogeneity $\mathrm{P}=0.04)$ or when evaluating with cessation as sustained abstinence (52 trials, total $\mathrm{n}=22,704$, OR $1.7295 \% \mathrm{CI}$, 1.54-1.93, $\mathrm{P}=<0.0001, \mathrm{I}^{2}=29.4 \%$, Heterogeneity $\mathrm{P}=$
0.02 ) or point prevalence ( 31 trials, $\mathrm{n}=10,686$, OR 1.53, 95\% CI, 1.30-1.81, P = 0.01, $\mathrm{I}^{2}=46 \%$, Heterogeneity $\mathrm{P}=$ $0.01)$. This was also consistent whether one evaluated NRT gum (33 trials, total $\mathrm{n}=12,245$, OR 1.60, 95\% CI, 1.37-1.86, $\mathrm{P}=<0.0001, \mathrm{I}^{2}=35.8 \%$, Heterogeneity $\mathrm{P}=$ 0.02 ) or NRT patch (23 trials, total $\mathrm{n}=11,108$, OR 1.63, $95 \% \mathrm{CI}, 1.41-1.89, \mathrm{P}=<0.0001, \mathrm{I}^{2}=12.3 \%$, Heterogeneity $\mathrm{P}=0.24)$.

Fifty-nine trials (total $\mathrm{n}=25,294$ ) provided sufficient details to determine short-term effects of NRT on smoking cessation, as determined at 3 months. The pooled OR of the 59 trials was 1.98 (95\% CI, 1.77-2.21, $\mathrm{P}=<0.0001, \mathrm{I}^{2}$ $=55.5 \%$, Heterogeneity $\mathrm{P}=<0.0001$, See Figure 3$)$. The superiority of NRT over controls was consistent whether one evaluated placebo-controlled trials (42 trials, total n $=19,216$, OR 2.11, 95\% CI, 1.86-2.40, P =< 0.0001, $\mathrm{I}^{2}=$ $57.6 \%$, Heterogeneity $\mathrm{P}=<0.001)$, sustained abstinence (41 trials, total $\mathrm{n}=19,854$, OR 2.04, 95\% CI, 1.80-2.31, 
Table I: Univariable meta-regression of NRT studies

\begin{tabular}{|c|c|c|c|c|c|c|c|}
\hline Covariates & $\begin{array}{c}\text { Point } \\
\text { estimate }\end{array}$ & $\begin{array}{l}\text { Lower } 95 \% \\
\text { limit }\end{array}$ & $\begin{array}{l}\text { Upper } 95 \% \\
\text { limit }\end{array}$ & OR & $\begin{array}{l}\text { Lower } 95 \% \\
\text { limit }\end{array}$ & $\begin{array}{c}\text { Upper } 95 \% \\
\text { limit }\end{array}$ & p-value \\
\hline Placebo & 0.13 & -0.03 & 0.31 & 1.13 & 0.97 & 1.36 & 0.12 \\
\hline $\begin{array}{l}\text { Sequence } \\
\text { generation }\end{array}$ & -0.15 & -0.32 & 0.006 & 0.86 & 0.72 & 1.00 & 0.06 \\
\hline $\begin{array}{l}\text { Allocation } \\
\text { concealment }\end{array}$ & -0.20 & -0.39 & 0.01 & 0.81 & 0.67 & 1.01 & 0.03 \\
\hline Gum & -0.18 & -0.32 & -0.02 & 0.83 & 0.72 & 0.98 & 0.02 \\
\hline Patch & 0.0007 & -0.15 & 0.16 & 1.00 & 0.86 & 1.17 & 0.99 \\
\hline $\mathrm{CO}$ & 0.52 & 0.24 & 0.80 & 1.68 & 1.27 & 2.22 & 0.0002 \\
\hline Cotinine & -0.21 & -0.37 & -0.05 & 0.81 & 0.69 & 0.95 & 0.007 \\
\hline Serum & -0.36 & -0.75 & 0.01 & 0.69 & 0.47 & 1.01 & 0.06 \\
\hline Urine & -0.36 & -0.70 & -0.02 & 0.69 & 0.49 & 0.98 & 0.03 \\
\hline Planned to quit & 0.12 & -0.02 & 0.27 & 1.12 & 0.98 & 1.30 & 0.10 \\
\hline
\end{tabular}

Legend. The point estimate and $95 \% \mathrm{Cls}$ estimate the unit change in the effect size, whenever the predicted covariate is present. The OR for the point estimates and $95 \% \mathrm{Cl}$ denote the likelihood of covariate affecting the trial effect size.

$\mathrm{P}=<0.0001, \mathrm{I}^{2}=58 \%$, Heterogeneity $\left.\mathrm{P}=<0.0001\right)$ or point prevalence at 3 months (21 trials, total $\mathrm{n}=6,453$, OR $1.78,95 \%$ CI, 1.47-2.14, $\mathrm{P}=<0.0001, \mathrm{I}^{2}=42.4$, Heterogeneity $\mathrm{P}=0.004)$. Studies assessing gum versus controls at 3 months ( 24 trials, total $\mathrm{n}=9,347$ ) yielded an OR of 1.71 (95\% CI, 1.41-2.07, $\mathrm{P}=<0.0001, \mathrm{I}^{2}=62 \%$, Heterogeneity $\mathrm{P}=<0.0001)$ and studies assessing patch versus controls ( 21 trials, total $\mathrm{n}=10,957)$ yielded an OR of 1.93 (95\% CI, 1.67-2.24, P =<0.0001, I2 = 35\%, Heterogeneity $\mathrm{P}=0.05)$.

\section{Bupropion}

We evaluated the effect of bupropion on smoking cessation relative to adequate controls at 1 year in 12 trials (total $\mathrm{n}=5,228$, See Figure 4). The pooled OR was 1.56 (95\% CI, 1.10-2.21, P = 0.01, $\mathrm{I}^{2}=71.5 \%$, Heterogeneity $\mathrm{P}$ $=<0.001)$. This effect was consistent whether examining placebo-controls ( 11 trials, total $\mathrm{n}=5,148$, OR $1.64,95 \%$ CI, 1.16-2.30, $\mathrm{P}=<0.001, \mathrm{I}^{2}=72 \%$, Heterogeneity $\mathrm{P}=$ $0.001)$, sustained abstinence (11 trials, total $\mathrm{n}=4,613$, OR 1.52, 95\% CI, 1.04-2.23, $\mathrm{P}=<0.0001, \mathrm{I}^{2}=73.6 \%$, Heterogeneity $\mathrm{P}=0.0001$ ), or point prevalence (10 trials, total $\mathrm{n}=4,845$, OR 1.56, 95\% CI, 1.13-2.16, $\mathrm{P}=<0.0001$, $\mathrm{I}^{2}=75.1 \%$, Heterogeneity $\left.\mathrm{P}=<0.0001\right)$.

When we evaluated the effect of bupropion on placebo at 3 months ( 11 trials, total $\mathrm{n}=5,148$ ), the OR was 2.13 (95\% CI, 1.72-2.64, $\mathrm{P}=<0.0001, \mathrm{I}^{2}=53.6 \%$, Heterogeneity $\mathrm{P}=0.01$, See Figure 5$)$. This effect was consistent across sustained abstinence measures ( 8 trials, total $\mathrm{n}=4,143$, OR 2.18, 95\% CI, 1.67-2.86, $\mathrm{P}=<0.0001, \mathrm{I}^{2}=63.5 \%$, Heterogeneity $\mathrm{P}=0.008$ ) and point prevalence measures (9 trials, total $\mathrm{n}=4,765$, OR 2.11, 95\% CI, 1.77-2.52, P $=<0.0001, \mathrm{I}^{2}=38.8 \%$, Heterogeneity $\left.\mathrm{P}=0.10\right)$.

\section{Varenicline}

We pooled 4 studies assessing the effect of varenicline versus placebo at 1 year (total $\mathrm{n}=2,528$, See Figure 6 ). The pooled OR is $2.96\left(95 \% \mathrm{CI}, 2.12-4.12, \mathrm{P}=<0.0001, \mathrm{I}^{2}=\right.$ $20.5 \%$, Heterogeneity $P=0.20)$. This effect was consistent with short-term cessation effects ( 4 trials, total $\mathrm{n}=2,528$, OR 3.75, 95\% CI, 2.65-5.30, $\mathrm{P}=<0.0001, \mathrm{I}^{2}=57.7 \%$, Heterogeneity $\mathrm{P}=0.06$, See Figure 7 ). We explained heterogeneity in this analysis through the inclusion of the dose-ranging studies[8,9].

\section{Comparisons}

Two trials evaluated the superiority of NRT versus bupropion at 1 year [82,97] (total $\mathrm{n}=548$, See Figure 8 ) and found a pooled OR of 1.14 (95\% CI, 0.20-6.42, P = 0.88, $\mathrm{I}^{2}=59 \%$, Heterogeneity $\mathrm{P}=0.11$. Only 1 trial provided details on cessation rates at 3 months and favored bupropion (OR 2.66, 95\% CI 1.70-4.15, $\mathrm{P}=<0.001$ )[82]. Three trials evaluated the effectiveness of varenicline versus bupropion at 1 year $[5,6,8]$ and yielded a pooled OR of 1.58 (95\% CI, 1.22-2.05, P = 0.001, $\mathrm{I}^{2}=0 \%$, Heterogeneity $\mathrm{P}=0.81$, See Figure 9$)$ in favor of varenicline. These same trials provided consistent data at 3 months (OR $1.61,95 \%$ CI, 1.16-2.21, P = 0.004, $\mathrm{I}^{2}=56.1 \%$, Heterogeneity $\mathrm{P}=0.10$, See Figure 10 ).

Using indirect comparisons[28], we found that bupropion was not superior to NRT when compared to a placebo control at 1 year (OR 0.92, 95\% CI 0.64-1.32, test for difference, $\mathrm{P}=0.65)$. This was similar for 3 -month data (OR 1.01, 95\% CI 0.79-1.29, test for difference 0.94). We found that varenicline was superior to NRT when compared to placebo controls (OR 1.66, 95\% CI, $1.17-2.36$, test for difference $P=0.004$, See Figure 11 ) or 


\begin{tabular}{|c|c|c|c|c|c|}
\hline \multirow[t]{2}{*}{ Study nar } & \multicolumn{5}{|c|}{ Statistics for each study } \\
\hline & \multicolumn{4}{|c|}{$\begin{array}{l}\text { Odds Lower Upper } \\
\text { ratiolimitlimitZ-Valuep-Value }\end{array}$} & \\
\hline Schneider N1 & 1.481 & 0.541 & 4.057 & 0.765 & 0.444 \\
\hline \multicolumn{2}{|c|}{ British Thoracic Societ $\emptyset .836$} & 0.533 & 1.312 & -0.777 & 0.437 \\
\hline Russell MAH & 2.026 & 1.379 & 2.978 & 3.595 & 0.000 \\
\hline Jarvik ME & 1.847 & 0.461 & 7.397 & 0.867 & 0.386 \\
\hline Fagerstrom $\mathrm{K}$ & 3.624 & 1.301 & 10.093 & 2.463 & 0.014 \\
\hline Hjalmarson A1 & 2.144 & 1.087 & 4.230 & 2.201 & 0.028 \\
\hline Hall S1 & 2.035 & 0.783 & 5.289 & 1.458 & 0.145 \\
\hline Clavel & 4.773 & 1.910 & 11.932 & 3.344 & 0.001 \\
\hline Hall S2 & 2.822 & 1.329 & 5.994 & 2.700 & 0.007 \\
\hline Sutton 1 & 5.313 & 0.701 & 40.255 & 1.617 & 0.106 \\
\hline Campbell L1 & 1.416 & 0.599 & 3.350 & 0.792 & 0.428 \\
\hline Sutton 2 & 2.703 & 0.509 & 14.357 & 1.167 & 0.243 \\
\hline Tonneson 1 & 2.124 & 0.928 & 4.858 & 1.784 & 0.074 \\
\hline Harackiewicz JM & 0.887 & 0.326 & 2.408 & -0.236 & 0.814 \\
\hline Blondal T1 & 1.850 & 0.989 & 3.460 & & 0.054 \\
\hline Hughes JR & 1.480 & 0.712 & 3.076 & & 0.294 \\
\hline Gilbert J & 0.858 & 0.300 & 2.453 & -0.285 & 0.776 \\
\hline Killen JD & 1.362 & 1.025 & 1.811 & & 0.033 \\
\hline Segnan $\mathrm{N}$ & 1.402 & 0.712 & 2.762 & 0.977 & 0.329 \\
\hline Tonneson 2 & 5.829 & 1.660 & 20.470 & 2.751 & 0.006 \\
\hline Campbell L2 & 0.977 & 0.497 & 1.919 & -0.068 & 0.946 \\
\hline Zelman DC & 1.460 & 0.679 & 3.140 & 0.969 & 0.332 \\
\hline Mcgovern PG & 1.168 & 0.704 & 1.937 & 0.600 & 0.548 \\
\hline Sutherland & 3.171 & 1.500 & 6.704 & & 0.003 \\
\hline Pirie PL & 1.299 & 0.810 & & & 0.278 \\
\hline Nebot M & 1.269 & 0.405 & & & 0.683 \\
\hline Tonne & 3.424 & 1.413 & 8.296 & 2.726 & 0.006 \\
\hline Sachs & 3.195 & 1.467 & 6.960 & & 0.003 \\
\hline Hurt RD & 2.298 & 1.199 & 4.407 & 2.505 & 0.012 \\
\hline Fowler G & 1.481 & 1.028 & 2.132 & 2.111 & 0.035 \\
\hline Hjalmarson A2 & 2.179 & 1.153 & 4.119 & 2.399 & 0.016 \\
\hline Niaura $R$ & 1.345 & 0.349 & 5.188 & 0.430 & 0.667 \\
\hline Fortmann SP & & 1.017 & & & 0.039 \\
\hline Korn & 0.943 & 0.415 & 2.144 & -0 . & 0.888 \\
\hline Dale LC & 1.403 & 0.472 & 4.175 & 0.609 & 0.543 \\
\hline Stapleton JA & 2.136 & 1.273 & 3.582 & 2.876 & 0.004 \\
\hline Herrera N & 3.404 & 1.689 & 6.861 & 3.426 & 0.001 \\
\hline Schneider N2 & 2.563 & 1.166 & 5.634 & 2.341 & 0.019 \\
\hline Puska P & 1.506 & 0.856 & 2.650 & 1.421 & 0.155 \\
\hline Campbell LA & 1.582 & 0.800 & 3.132 & 1.318 & 0.188 \\
\hline Hall SM & 0.869 & 0.461 & 1.636 & -0.436 & 0.663 \\
\hline Leischow SJ & 2.121 & 0.767 & 5.869 & & 0.148 \\
\hline Cin & 3 & 0.712 & 6. & 55 & 0.175 \\
\hline Schn & 1.753 & 0.733 & 4.191 & 1.261 & 0.207 \\
\hline Paoletti P & 4.349 & 1.486 & 12.729 & 2.683 & 0.007 \\
\hline Killen JD & 1.107 & 0.593 & 2.067 & 0.318 & 0.750 \\
\hline Blondal T & 1.695 & 0.775 & 3.705 & 1.322 & 0.186 \\
\hline Richmond RL & 2.305 & 1.165 & 4.561 & 2.399 & 0.016 \\
\hline Hjalmarson A3 & 1.844 & 1.007 & 3.376 & 1.983 & 0.047 \\
\hline Daughton D & 1.816 & 0.943 & 3.498 & 1.785 & 0.074 \\
\hline & 4.143 & 0.999 & 17.178 & 1.959 & 0.050 \\
\hline Niaura $R$ & 0.688 & 0.224 & 2.107 & -0 & 0.512 \\
\hline Tonn & 1.498 & 1.147 & 1.955 & 2.969 & 0.003 \\
\hline & 1.830 & 0.828 & 4.047 & 1.493 & 0.135 \\
\hline Blondal T 2 & 3.034 & 1.500 & 6.138 & 3.087 & 0.002 \\
\hline Bolliger CT & 1.362 & 0.627 & 2.959 & 0.781 & 0.435 \\
\hline Garvey AJ & 2.487 & 1.426 & 4.337 & 3.209 & 0.001 \\
\hline Wisborg & 1.086 & 0.540 & 2.183 & 0.231 & 0.817 \\
\hline Tonneson 5 & 0.673 & 0.185 & & & 0.548 \\
\hline Wallstrom M & 1.629 & 0.854 & & & 0.138 \\
\hline Boh & 1.488 & 0.875 & 2.530 & 1.467 & 0.142 \\
\hline Glover ED & 2.039 & 0.959 & 4.336 & 1.851 & 0.064 \\
\hline Shiffman & 2.279 & 1.692 & 3.070 & 5.419 & 0.000 \\
\hline Hand S & 1.080 & 0.525 & 2.226 & 0.210 & 0.834 \\
\hline Wennike $\mathrm{P}$ & 3.128 & 1.365 & 7.168 & 2.695 & 0.007 \\
\hline Glavas D & 1.579 & 0.614 & 4.063 & 0.947 & 0.344 \\
\hline Molyneux A & 3.395 & 1.178 & 9.780 & 2.264 & 0.024 \\
\hline Cooper TV & 1.168 & 0.560 & 2.438 & 0.415 & 0.678 \\
\hline Batra A & 2.920 & 1.264 & 6.744 & 2.509 & 0.012 \\
\hline \multirow[t]{2}{*}{ Swanson NA } & 0.683 & 0.162 & 2.868 & -0.521 & 0.602 \\
\hline & 1.713 & 1.556 & 1.886 & 10.988 & 0.000 \\
\hline
\end{tabular}
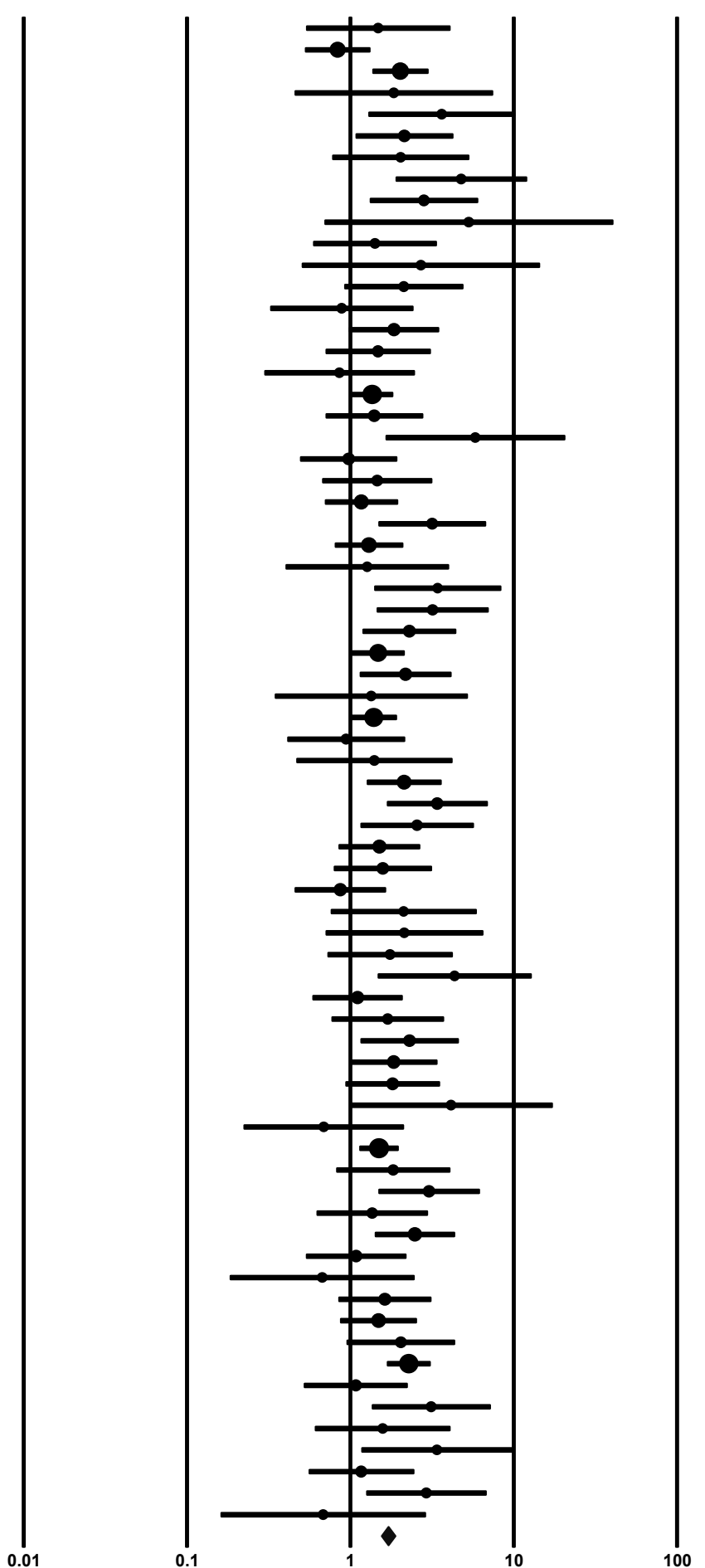

Favours ControlFavours NRT

Figure 2

Random Effects Meta-Analysis. NRT vs. Controls at 12 months. $\left.\right|^{2}=26.5 \%$. 


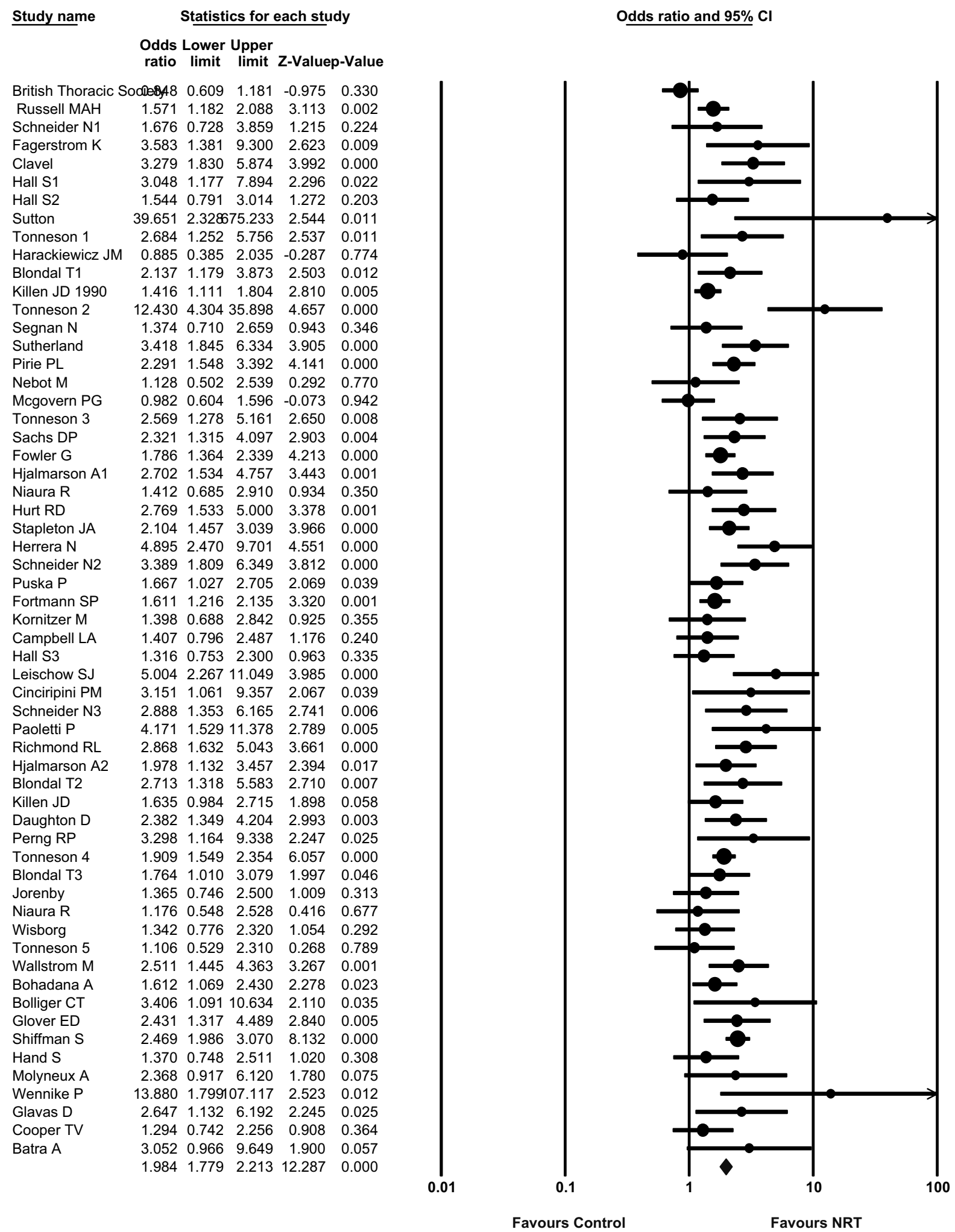

Figure 3

Random Effects Meta-Analysis. NRT vs. Controls at 3 months. $1^{2}=55.5 \%$. 


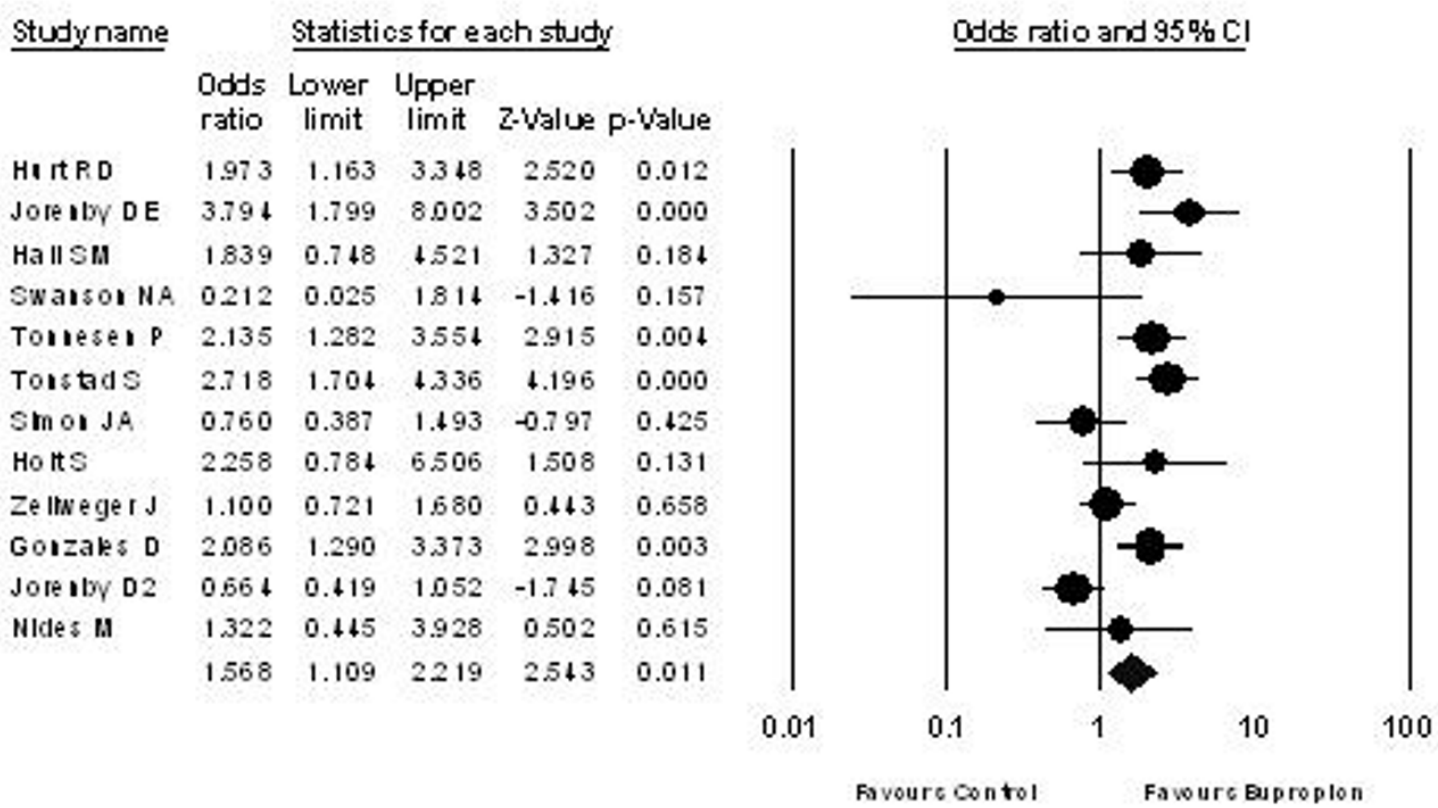

Figure 4

Random Effects Meta-Analysis. Bupropion vs. Controls at 12 months. $1^{2}=71.5 \%$.

\begin{tabular}{lcccccc} 
Study name & & \multicolumn{5}{c}{ Statistics for each study } \\
\cline { 1 - 6 } & & $\begin{array}{c}\text { Odds } \\
\text { ratio }\end{array}$ & $\begin{array}{c}\text { Lower } \\
\text { limit }\end{array}$ & $\begin{array}{c}\text { Upper } \\
\text { limit }\end{array}$ & Z-Value & p-Value \\
Hurt RD & 2.160 & 1.315 & 3.550 & 3.041 & 0.002 \\
Jorenby DE & 3.637 & 2.078 & 6.367 & 4.521 & 0.000 \\
Hall SM & 2.939 & 1.429 & 6.045 & 2.930 & 0.003 \\
Tonnesen P & 2.183 & 1.429 & 3.335 & 3.613 & 0.000 \\
Tonstad S & 2.898 & 1.965 & 4.274 & 5.367 & 0.000 \\
Simon JA & 1.487 & 0.898 & 2.464 & 1.540 & 0.123 \\
Holt S & 3.781 & 1.583 & 9.031 & 2.994 & 0.003 \\
Zellweger J & 1.132 & 0.781 & 1.640 & 0.653 & 0.514 \\
Gonzales D & 1.940 & 1.348 & 2.792 & 3.565 & 0.000 \\
Jorenby D2 & 1.990 & 1.385 & 2.860 & 3.722 & 0.000 \\
Nides M & 2.094 & 1.017 & 4.314 & 2.005 & 0.045 \\
& 2.132 & 1.720 & 2.642 & 6.909 & 0.000
\end{tabular}

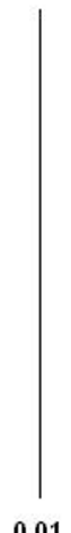

0.01
Odds ratio and $95 \% \mathrm{Cl}$
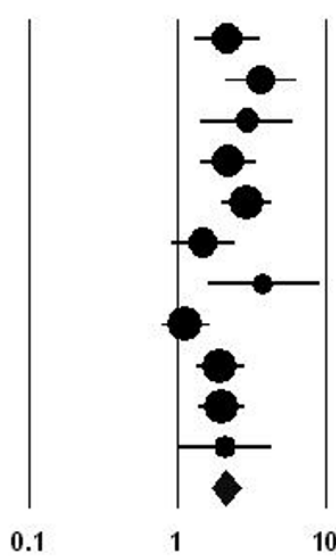

10

100

Favours Control

Favours Bupropion

Figure 5

Random Effects Meta-Analysis. Bupropion vs. Controls at 3 months. $I^{2}=53.6 \%$. 


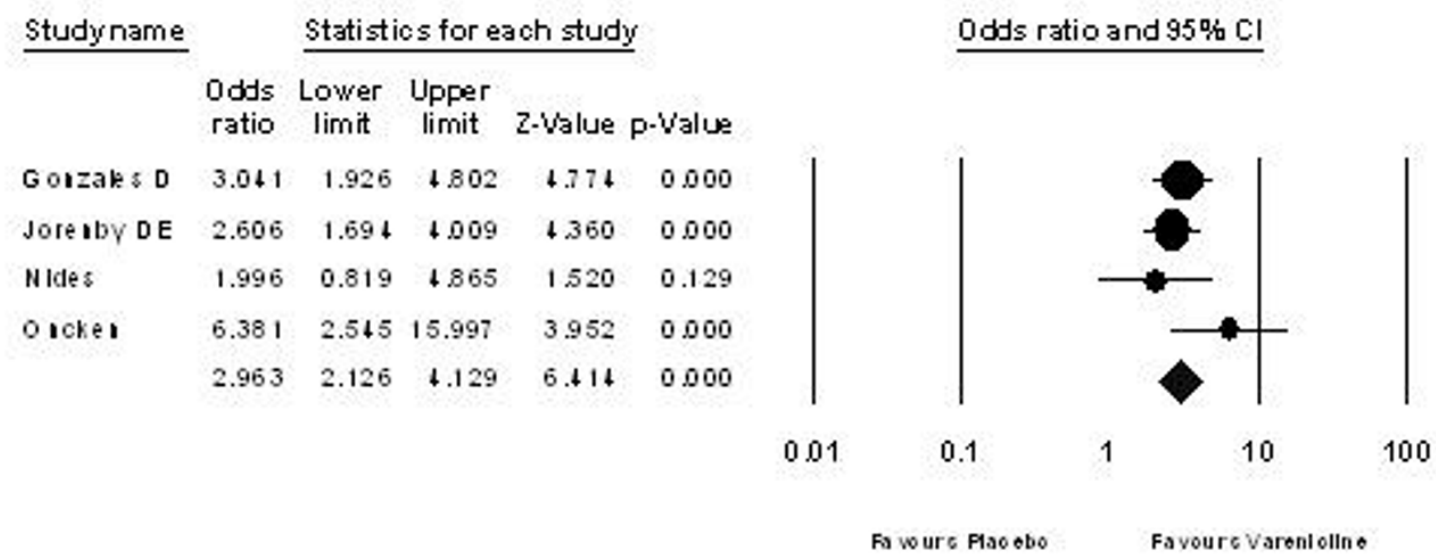

\section{Figure 6}

Random Effects Meta-Analysis. Varenicline vs. Placebo at 12 months. ${ }^{2}=20.5 \%$.

to all controls at 1 year (OR 1.73, 95\% CI 1.22-2.45, test for difference $\mathrm{P}=0.001)$. This was also the case when we examined 3-month data for placebo controls (OR 1.78, $95 \% \mathrm{CI}, 1.23-2.57$, test for difference $\mathrm{P}=0.002$, See Figure 12) or all controls (OR 1.89, 95\% CI, 1.31-2.73, test for difference $\mathrm{P}=0.0006$ ).

\section{Meta-regression}

We anticipated variable between-study heterogeneity, considering the interventions used, methodological issues and measurement tools. Table 1 displays the covariates predicting heterogeneity in the primary outcomes of the NRT analysis using meta-regression. In this analysis, significant predictors of heterogeneity included: allocation concealment, use of NRT gum; and, methods of chemical confirmation ( $\mathrm{CO}$, cotinine, and urine markers). Using sensitivity analysis, only studies ( $n=3[49,53,71])$ using urine as a marker were significantly different from the pooled estimate $(P=0.03)$, however, all but 1 of these studies also used $\mathrm{CO}$ as a chemical marker $(\mathrm{P}=0.5)[49]$.

We additionally examined covariates in the bupropion trials (See Table 2). We found sequence generation was a significant contributor to heterogeneity. In addition, the chemical marker covariates contributed to heterogeneity. We did not conduct a meta-regression on the varenicline studies, given the small number of studies.

\section{Adverse events}

Additional File 1 displays the common adverse events associated with NRT. Inadequate detail was provided for pooling. For NRT trials, we found that the following

\begin{tabular}{llrrrrr} 
Study name & \multicolumn{5}{c}{ Statistics for each study } \\
\cline { 1 - 4 } & $\begin{array}{l}\text { Odds } \\
\text { ratio }\end{array}$ & $\begin{array}{c}\text { Lower } \\
\text { limit }\end{array}$ & $\begin{array}{c}\text { Upper } \\
\text { limit }\end{array}$ & Z-Value & p-Value \\
Gonzales D & 3.650 & 2.578 & 5.168 & 7.301 & 0.000 \\
Jorenby DE & 3.664 & 2.580 & 5.204 & 7.256 & 0.000 \\
Nides & 2.136 & 1.141 & 4.001 & 2.371 & 0.018 \\
Oncken & 6.664 & 3.786 & 11.728 & 6.576 & 0.000 \\
& 3.750 & 2.651 & 5.303 & 7.473 & 0.000
\end{tabular}

\section{Figure 7}

Random Effects Meta-Analysis. Varenicline vs. Placebo at 3 months. ${ }^{2}=57.7 \%$. 


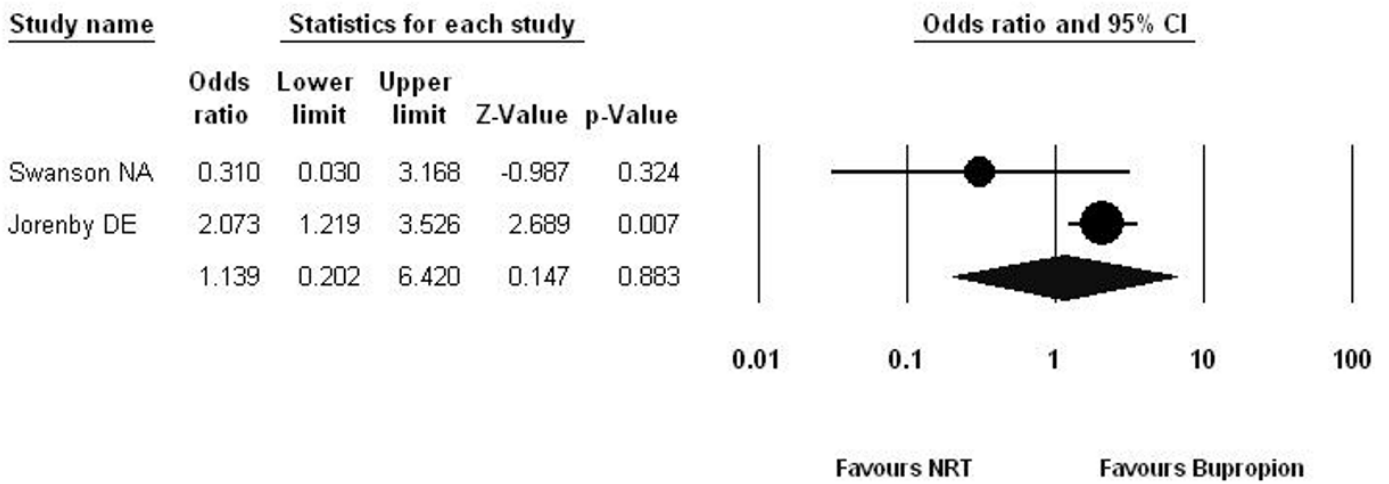

\section{Figure 8}

Random Effects Meta-Analysis. NRT vs. Bupropion at 12 months. $I^{2}=59 \%$.

adverse events were reported significantly more often in active groups than control groups: mouth or throat irritation $(\mathrm{n}=12)$; skin irritation $(\mathrm{n}=11)$; nausea/vomiting $(\mathrm{n}$ $=10)$; coughing $(\mathrm{n}=9)$; hiccoughs $(\mathrm{n}=6)$; dyspepsia $(\mathrm{n}=$ $4)$; watering of eyes $(n=3)$; headaches $(n=3)$; heart palpitations $(n=3)$; sneezing $(n=3)$; sleep disturbances and dream abnormalities $(\mathrm{n}=2)$; insomnia $(\mathrm{n}=2)$; rhinitis $(\mathrm{n}$ $=2)$; vertigo $(n=1)$; taste disturbances $(n=1)$ and muscle aches $(\mathrm{n}=1)$.

For bupropion trials, the following adverse events were reported significantly more in the active groups than control groups: dry mouth (9 trials[5,6,8,82,102-106], $\mathrm{n}=$ 4,885, OR 1.90, 95\% CI, 1.50-2.42, P =<0.0001); insomnia (10 trials[5,6,8,82,101-106], $\mathrm{n}=4,775$, OR 2.02, 95\% CI, 1.53-2.68, $\mathrm{P}=<0.0001$ ); gastrointestinal upset ( 7 trials[5,6,8,82,104-106], $\mathrm{n}=4,026$, OR 1.34, 95\% CI, $1.06-$ $1.70, \mathrm{P}=0.01$ ) and constipation ( 6 trials $[5,6,8,104-106]$, $\mathrm{n}=3,622$, OR 2.48, 95\% CI, 1.62-3.80, $\mathrm{P}=<0.0001)$. Other severe events associated with trial participants in the active arms were: septic shock; grand mal seizure; sleep disorders; and anxiety. These were single cases and did not achieve significance.

For varenicline trials, the following adverse events were reported significantly more often than in the placebo groups: nausea (4 trials $[5,6,8,9], \mathrm{n}=2,506$, OR 3.17, 2.35-4.29, $\mathrm{P}=<0.0001)$; flatulence ( 2 trials $[5,9], \mathrm{n}=$ 1,323, OR 2.04, 95\% CI, 1.16-3.57, P = 0.01); and, constipation ( 4 trials $[5,6,8,9], \mathrm{n}=2,506$, OR $2.57,95 \% \mathrm{CI}$, 1.21-5.45, $\mathrm{P}=<0.0001)$. Other, severe events that occurred in the active groups included: atrial fibrillation, pneumonia, possible stroke, chest pain, and elevated blood pressure. These were, however, single cases and did not achieve significance.

\begin{tabular}{|c|c|c|c|c|c|}
\hline \multirow[t]{2}{*}{ Study name } & \multirow[b]{2}{*}{$\begin{array}{l}\text { Odds } \\
\text { ratio }\end{array}$} & \multicolumn{3}{|c|}{ Statistics for each study } & \multirow[b]{2}{*}{ p-Value } \\
\hline & & $\begin{array}{c}\text { Lower } \\
\text { limit }\end{array}$ & $\begin{array}{c}\text { Upper } \\
\text { limit }\end{array}$ & Z-Value & \\
\hline Gonzales D & 1.458 & 0.990 & 2.149 & 1.907 & 0.057 \\
\hline Jorenby DE & 1.741 & 1.177 & 2.575 & 2.777 & 0.005 \\
\hline \multirow[t]{2}{*}{ Nides M } & 1.510 & 0.681 & 3.346 & 1.014 & 0.3 \\
\hline & 1.583 & 1.220 & 2.053 & 3.459 & 0.00 \\
\hline
\end{tabular}

\section{Figure 9}

Random Effects Meta-Analysis. Varenicline vs. Bupropion at 12 months. $1^{2}=0 \%$. 


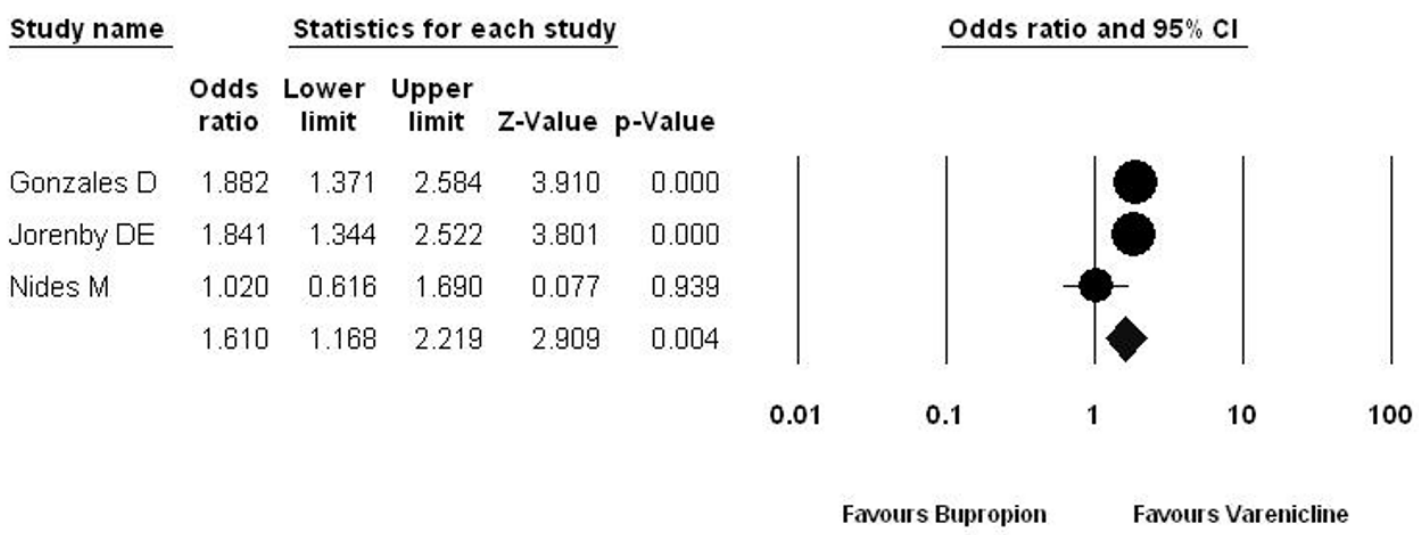

Figure 10

Random Effects Meta-Analysis. Varenicline vs. Bupropion at 3 months. $1^{2}=56.1 \%$.

\section{Discussion}

In this study we present the results of three main metaanalyses examining the effectiveness of various pharmacological strategies to improve smoking cessation. Our primary meta-analyses specifically examined the effectiveness of NRT versus control, bupropion versus control, and varenicline versus bupropion or placebo. We demonstrated the consistent effectiveness of each intervention in short-term and long-term smoking cessation.

Our findings confirm the effectiveness of two established pharmacological therapies, NRT and bupropion, to improve cessation rates. The primary new information from this review is the evidence of effectiveness of varenicline. This modality of treatment was identified to be more effective than placebo, bupropion and in indirect comparisons with NRT. However, this meta-analysis con-

\section{Study name}

$\begin{array}{llll} & \begin{array}{c}\text { Odds } \\ \text { ratio }\end{array} & \begin{array}{c}\text { Lower } \\ \text { limit }\end{array} & \begin{array}{c}\text { Upper } \\ \text { limit }\end{array} \\ \text { NRT vs Placebo } & 1.780 & 1.595 & 1.986 \\ \text { Varenicline vs Placebo } & 2.960 & 2.123 & 4.126 \\ \text { In-direct comparison } & 1.66 & 1.17 & 2.36\end{array}$

sisted of the fewest studies and although the studies were well reported and apparently well conducted, further studies comparing varenicline versus either NRT or bupropion will strengthen inferences about the superiority of this intervention. Nevertheless the initial findings are suggestive of the potential superiority of this latest pharmacological intervention.

This review has several strengths and some limitations that deserve mention. The strengths of this review include the comprehensive search strategy that improved the likelihood of identifying all relevant studies. Duplicate extraction of data reduced the potential for bias in this component of the synthesis process. By limiting this review to randomized trials we ensured that the included studies would have reduced likelihood of systematic error and therefore have high internal validity. Our use of meta-

\section{Odds ratio and $95 \% \mathrm{Cl}$}

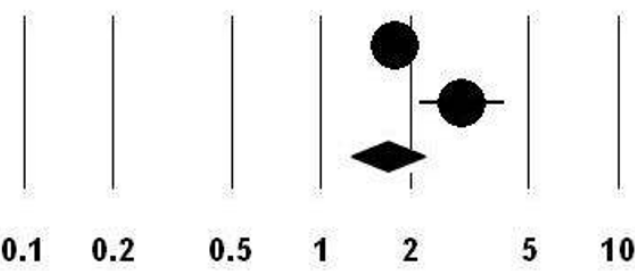

Favours NRT Favours Varenicline

\section{Figure I I}

Indirect comparison between Varenicline and NRT vs Placebo at $\mathbf{2}$ months. Bucher et al. indirect comparison methods [28]. 
Study name

$\begin{array}{lllll} & \begin{array}{c}\text { Odds } \\ \text { ratio }\end{array} & \begin{array}{c}\text { Lower } \\ \text { limit }\end{array} & \begin{array}{c}\text { Upper } \\ \text { limit }\end{array} \\ \text { Varenicline vs Placebo } & 3.750 & 2.652 & 5.303 \\ \text { NRT vs Placebo } & 2.110 & 1.856 & 2.398 \\ \text { In-direct comparison } & 1.78 & 1.23 & 2.57\end{array}$

Odds ratio and $95 \% \mathrm{Cl}$

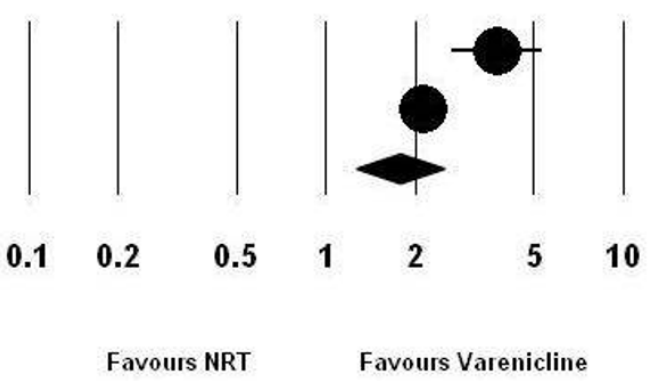

Figure 12

Indirect comparison between Varenicline and NRT vs Placebo at 3 months. Bucher et al. indirect comparison methods [28].

regression to identify sources of heterogeneity in the meta-analyses is a strength and demonstrated that several of the a priori chosen covariates were predictors of heterogeneity. While our primary endpoint was one-year cessation, as defined by the study, we evaluated the robustness of these findings through multiple sensitivity analyses which included one-year sustained cessation, a strong predictor of sustained smoking cessation[108,109]. Unlike some meta-analyses and systematic reviews of smoking cessation therapies [3,4,12-19], we included only studies that chemically confirmed the cessation of smoking at the specific time-points. This is a strength of our analysis as we demonstrated that even the different chemical markers for cessation can contribute greatly to between-study differences. Our meta-regression analysis indicates that the type of chemical markers employed yields greater variability of effects between studies over more commonly investigated covariates, such as methodological issues[110].

Limitations of this meta-analysis include the potential for publication bias, specifically the possibility that small negative studies would not be published. We included only published trials so it is possible that other trials have been conducted and never published. However, it is unlikely that the presence of these studies would have altered the findings of the NRT meta-analysis given the large number of studies included. Similarly, since varenicline is a new agent and the studies synthesized were the first clinical trials evaluating its effectiveness, it is improbable other negative studies exist. We also limited our search to English language databases (although we would include non-English articles if identified) so the possibility of quality studies in other languages does exist. We used both direct and indirect comparisons to evaluate the relative effectiveness of agents. Head-to-head trials provide the strongest inferences regarding intervention superiority[27]. However, in the absence of head-to-head trials, specifically NRT versus varenicline, we conducted indirect comparisons. We used the indirect comparison method proposed by Bucher et al., which respects the principle of randomization between trials [28]. The utility of indirect comparisons is debatable and some evidence suggests that indirect comparisons may provide misleading measures of superiority, although the direction of bias

Table 2: Univariable meta-regression of bupropion studies.

\begin{tabular}{|c|c|c|c|c|c|c|c|}
\hline Covariates & $\begin{array}{c}\text { Point } \\
\text { estimate }\end{array}$ & $\begin{array}{l}\text { Lower } 95 \% \\
\text { limit }\end{array}$ & $\begin{array}{l}\text { Upper 95\% } \\
\text { limit }\end{array}$ & OR & $\begin{array}{l}\text { Lower 95\% } \\
\text { limit }\end{array}$ & $\begin{array}{c}\text { Upper } 95 \% \\
\text { limit }\end{array}$ & p-value \\
\hline Placebo & 2.00 & -0.14 & 4.15 & 7.38 & 0.86 & 63.4 & 0.07 \\
\hline $\begin{array}{l}\text { Sequence } \\
\text { generation }\end{array}$ & -0.46 & -0.84 & -0.08 & 0.63 & 0.43 & 0.92 & 0.01 \\
\hline $\begin{array}{l}\text { Allocation } \\
\text { concealment }\end{array}$ & -0.20 & -0.55 & 0.14 & 0.81 & 0.57 & 1.15 & 0.25 \\
\hline $\mathrm{CO}$ & 0.76 & 0.06 & 1.46 & 2.13 & 1.06 & 4.30 & 0.03 \\
\hline Cotinine & -0.76 & -1.46 & -0.06 & 0.46 & 0.23 & 0.94 & 0.03 \\
\hline Planned to quit & 0.13 & -0.22 & 0.48 & 1.13 & 0.80 & $1.6 \mid$ & 0.46 \\
\hline
\end{tabular}

Legend. The point estimate and $95 \% \mathrm{Cls}$ estimate the unit change in the effect size, whenever the predicted covariate is present. The OR for the point estimates and $95 \% \mathrm{Cl}$ denote the likelihood of covariate affecting the trial effect size. 
cannot be assumed until direct comparison trials have been completed[111]. Figure 8 displays an interesting methodologic outcome related to choosing randomeffects models over a fixed-effects model for meta-analyses. The pooled OR of the two head-to-head trials is a nonsignificant difference of 1.14 (95\% CI, 0.20-6.42, P = 0.88 ) when using the random effects model, as planned in our protocol $[82,97]$. However, had we used a fixed effects model, this would have resulted in a significant pooled OR of 1.84 (95\% CI, 1.11-3.06, P=0.02). We believe that this is due to the very small number of trials included ( $\mathrm{n}$ $=2$ ) $[82,97]$, where one trial is much larger than the other (n of 488 compared to an n of 60 ). Finally, our meta-analyses did not examine in detail other aspects of therapy, such as compliance and tolerance, which may influence real life clinical effectiveness.

There are several important implications of these metaanalyses. Identifying effective mechanisms to improve smoking cessation is essential. Smoking is the number one preventable cause of death in the world[1]. Approximately one out of every two long-term smokers will die of a smoking related death[112]. Furthermore, smoking cessation has been clearly demonstrated to reduce the likelihood of future morbidity. Smoking cessation in individuals with chronic obstructive lung disease reduces the deterioration of $\mathrm{FEV}_{1}[113]$. Smoking cessation also reduces the likelihood of developing several smoking related cancers[109]. Smoking cessation in patients with coronary artery disease has been consistently shown to be associated with dramatically improved survival. Observational studies demonstrate that smokers who quit smoking after a myocardial infarction have mortality rates approximately $40 \%$ less than those that continue to smoke, suggesting that smoking cessation could be one of the most effective therapies to improve survival in this group of patients[114]. Former smokers have also been demonstrated to have considerably lower rates of cerebrovascular disease than ongoing smokers[115].

\section{Conclusion}

The findings of this review point the direction for future studies. It is interesting that the three modalities examined have distinct biological mechanisms of action. NRT presumably works by reducing symptoms of nicotine withdrawal, thereby increasing the likelihood of smoking cessation. Bupropion is a weak dopamine and nor-epinephrine reuptake inhibitor. One of the primary symptoms of smoking cessation has been depressive symptoms and it has been hypothesized that smokers may be increasing central dopamine levels by reducing monoamine oxidase inhibitor activity[116]. The mechanism of action of bupropion, therefore, may be to maintain central levels of dopamine through the process of cessation, although its effectiveness has been identified to be independent of symptoms of depression[102]. Varenicline is a nicotinic acetylcholine receptor partial agonist. The authors of one the studies demonstrating its efficacy comment that "Partial agonists at this (receptor) could stimulate the release of sufficient dopamine to reduce craving and withdrawal while simultaneously acting as a partial antagonist by blocking the binding and consequent reinforcing effects of smoked nicotine[5]." Regardless of the exact mechanism of action of the three modalities, it is clear they are distinct and suggest the possibility of combination therapy or therapy targeted on the particular type of symptoms experienced during cessation. Future studies could examine these options, given that despite the effectiveness of these therapies rates of smoking remain high at one year in the treatment groups [82]. Furthermore, given the benefits described of smoking cessation as secondary prevention, the use of these cessation modalities in patients with active smoking related disease warrants further study [117]. Future studies should further examine the safety and effectiveness in reducing morbidity and mortality of all three of these modalities in patients with active smoking related disease.

\section{Competing interests}

This study was supported by an unrestricted educational grant from Pfizer Global Solutions and the Canadian Institutes of Health Research (CIHR). The terms of the support from Pfizer and CIHR included freedom for authors to reach their own conclusions, and an absolute right to publish the results of their research, irrespective of any conclusions reached.

\section{Authors' contributions}

PW conducted the systematic searches, abstracted data, analyzed the data and contributed to the manuscript.

PD abstracted data, analyzed the data and contributed to the manuscript.

KW conceived the study, abstracted data, analyzed the data and contributed to the manuscript.

EM conceived the study, conducted the searches, abstracted data, analyzed the data and contributed to the manuscript.

All authors have read and approved the final manuscript.

\section{Additional material}

\section{Additional File 1}

Characteristics of NRT RCTs. Word file displays specific study details Click here for file

[http://www.biomedcentral.com/content/supplementary/14712458-6-300-S1.doc] 


\section{Additional File 2 \\ Characteristics of Bupropion RCTs. Word file displays specific study details \\ Click here for file \\ [http://www.biomedcentral.com/content/supplementary/1471- \\ 2458-6-300-S2.doc] \\ Additional File 3 \\ Characteristics of Varenicline RCTs. Word file displays specific study details \\ Click here for file \\ [http://www.biomedcentral.com/content/supplementary/1471- 2458-6-300-S3.doc]}

\section{Acknowledgements}

The contribution of Gordon Guyatt is gratefully acknowledged. KW and EM are supported by the Canadian Institutes of Health Research (CIHR). Thanks to Pearl Raju for information systems assistance.

\section{References}

I. Peto R, Lopez AD, Boreham J, Thun M, Heath C Jr, Doll R: Mortality from smoking worldwide. Br Med Bull 1996, 52: I2-2I.

2. Law M, Tang JL: An analysis of the effectiveness of interventions intended to help people stop smoking. Arch Intern Med 1995, I55:1933-1941

3. Silagy C, Lancaster T, Stead L, Mant D, Fowler G: Nicotine replacement therapy for smoking cessation. Cochrane Database Syst Rev 2004:CD000146.

4. Hughes J, Stead L, Lancaster T: Antidepressants for smoking cessation. Cochrane Database Syst Rev 2004:CD00003I.

5. Gonzales D, Rennard SI, Nides M, Oncken C, Azoulay S, Billing CB Watsky EJ, Gong J, Williams KE, Reeves KR: Varenicline, an alpha4beta2 nicotinic acetylcholine receptor partial agonist, vs sustained-release bupropion and placebo for smoking cessation: a randomized controlled trial. JAMA 2006, 296:47-55.

6. Jorenby DE, Hays JT, Rigotti NA, Azoulay S, Watsky EJ, Williams KE, Billing CB, Gong J, Reeves KR: Efficacy of varenicline, an alpha4beta2 nicotinic acetylcholine receptor partial agonist, vs placebo or sustained-release bupropion for smoking cessation: a randomized controlled trial. JAMA 2006, 296:56-63.

7. Tonstad S, Tonnesen P, Hajek P, Williams KE, Billing CB, Reeves KR: Effect of maintenance therapy with varenicline on smoking cessation: a randomized controlled trial. JAMA 2006, 296:64-7I

8. Nides M, C O, Gonzales D, Rennard SI, Watsky El, Anziano R, Reeves $\mathrm{KR}$ : Smoking cessation with varenicline, a selective alpha4beta2 nicotinic receptor partial agonist: results from a 7-week, randomized, placebo- and bupropion controlled trial with I-year follow-up. Arch Intern Med 2006, I 66: I 56 I-I568.

9. Oncken C, Gonzales D, Nides M, Rennard S, Watsky E, Billing CB Anziano R, Reeves KR: Efficacy and safety of the novel selective nicotinbic acetylcholine receptor partial agonist, varenicline, for smoking cessation. Arch Intern Med 2006, I 66: I 57| - I 577.

10. Ruth KJ, Neaton JD: Evaluation of two biological markers of tobacco exposure. MRFIT Research Group. Prev Med 199I, 20:574-589.

II. Benowitz NL: Measurement in the Analysis and Treatment of Smoking Behaviour Edited by: Grubowski J, Bell CS. NIDA Research Monograph 48. U.S. Department of Health and Human Services, Public Health Service, Alcohol, Drug Abuse, and Mental Health Administration, National Institute on Drug Abuse; 1983:3-26.

12. Lancaster T, Silagy C, Fowler G: Training health professionals in smoking cessation. Cochrane Database Syst Rev 2000:CD0002 I4.

13. Silagy C: Physician advice for smoking cessation. Cochrane Database Syst Rev 2000:CD000165.

14. Silagy C, Lancaster T, Stead L, Mant D, Fowler G: Nicotine replacement therapy for smoking cessation. Cochrane Database Syst Rev 2001:CD000146.
I5. Silagy C, Lancaster T, Stead L, Mant D, Fowler G: Nicotine replacement therapy for smoking cessation. Cochrane Database Syst Rev 2002:CD000146.

16. Silagy C, Mant D, Fowler G, Lancaster T: Nicotine replacement therapy for smoking cessation. Cochrane Database Syst Rev 2000:CD000I46.

17. Silagy C, Mant D, Fowler G, Lancaster T: Nicotine replacement therapy for smoking cessation. Cochrane Database Syst Rev 2000:CD000146.

18. Stead LF, Lancaster T, Silagy CA: Updating a systematic review what difference did it make? Case study of nicotine replacement therapy. BMC Med Res Methodol 200I, I: I0.

19. Hughes JR, Stead LF, Lancaster T: Antidepressants for smoking cessation. Cochrane Database Syst Rev 2002:CD00003I.

20. NICE: TA39 Smoking cessation - bupropion and nicotine replacement therapy: Guidance. Issue Date: March 2002 Review Date: March 2005. [http://www.nice.org.uk/30590]

21. Meade MO, Guyatt GH, Cook RJ, Groll R, Kachura JR, Wigg M, Cook DJ, Slutsky AS, Stewart TE: Agreement between alternative classifications of acute respiratory distress syndrome. $\mathrm{Am} J$ Respir Crit Care Med 200I, I 63:490-493.

22. Sheehe PR: Combination of log relative risk in retrospective studies of disease. Am \& Public Health Nations Health 1966, 56: $1745-1750$.

23. Fleiss JL: The statistical basis of meta-analysis. Stat Methods Med Res 1993, 2:121-145.

24. DerSimonian R, Laird N: Meta-analysis in clinical trials. Control Clin Trials 1986, 7:I77-I88.

25. Higgins JP, Thompson SG: Quantifying heterogeneity in a metaanalysis. Stat Med 2002, 21 : I539-I558.

26. Thompson SG, Higgins JP: How should meta-regression analyses be undertaken and interpreted? Stat Med 2002, 21:1559-I573.

27. McAlister FA, Laupacis A, Wells GA, Sackett DL: Users' Guides to the Medical Literature: XIX. Applying clinical trial results B. Guidelines for determining whether a drug is exerting (more than) a class effect. JAMA 1999, 282: |37|-|377.

28. Bucher HC, Guyatt GH, Griffith LE, Walter SD: The results of direct and indirect treatment comparisons in meta-analysis of randomized controlled trials. I Clin Epidemiol 1997 50:683-691.

29. Yusuf S, Peto R, Lewis J, Collins R, Sleight P: Beta blockade during and after myocardial infarction: an overview of the randomized trials. Prog Cardiovasc Dis 1985, 27:335-371.

30. Comparison of four methods of smoking withdrawal in patients with smoking related diseases. Report by a subcommittee of the Research Committee of the British Thoracic Society. Br Med J (Clin Res Ed) 1983, 286:595-597.

31. Russell MA, Merriman R, Stapleton J, Taylor W: Effect of nicotine chewing gum as an adjunct to general practitioner's advice against smoking. $\mathrm{Br}$ Med J (Clin Res Ed) I983, 287: I782-I785.

32. Schneider NG, Jarvik ME, Forsythe AB, Read LL, Elliott ML, Schweiger $A$ : Nicotine gum in smoking cessation: a placebo-controlled, double-blind trial. Addict Behav 1983, 8:253-26I.

33. Fagerstrom KO: Effects of nicotine chewing gum and follow-up appointments in physician-based smoking cessation. Prev Med 1984, 13:517-527.

34. Hjalmarson Al: Effect of nicotine chewing gum in smoking cessation. A randomized, placebo-controlled, double-blind study. JAMA 1984, 252:2835-2838.

35. Jarvik ME, Schneider NG: Degree of addiction and effectiveness of nicotine gum therapy for smoking. Am J Psychiatry 1984 | 41:790-79|.

36. Clavel F, Benhamou S, Company-Huertas A, Flamant R: Helping people to stop smoking: randomised comparison of groups being treated with acupuncture and nicotine gum with control group. Br Med J (Clin Res Ed) 1985, 29 I: I 538-I539.

37. Hall SM, Tunstall C, Rugg D, Jones RT, Benowitz N: Nicotine gum and behavioral treatment in smoking cessation. J Consult Clin Psychol 1985, 53:256-258.

38. Campbell IA, Lyons E, Prescott RJ: Stopping smoking. Do nicotine chewing-gum and postal encouragement add to doctors' advice. Practitioner 1987, 23 I: I |4-1।7.

39. Hall SM, Tunstall CD, Ginsberg D, Benowitz NL, Jones RT: Nicotine gum and behavioral treatment: a placebo controlled trial. Consult Clin Psychol 1987, 55:603-605. 
40. Sutton S, Hallett R: Randomized trial of brief individual treatment for smoking using nicotine chewing gum in a workplace setting. Am J Public Health 1987, 77:1210-121 I.

4I. Harackiewicz JM, Blair LW, Sansone C, Epstein JA, Stuchell RN: Nic otine gum and self-help manuals in smoking cessation: an evaluation in a medical context. Addict Behav 1988, I 3:3 19-330.

42. Sutton S, Hallett R: Smoking intervention in the workplace using videotapes and nicotine chewing gum. Prev Med 1988 1 7:48-59.

43. Tonnesen P, Fryd V, Hansen M, Helsted J, Gunnersen AB, Forchammer $H$, Stockner $M$ : Effect of nicotine chewing gum in combination with group counseling on the cessation of smoking. $N$ Engl J Med 1988, 3 18:15-18.

44. Blondal T: Controlled trial of nicotine polacrilex gum with supportive measures. Arch Intern Med 1989, I49:1818-182I.

45. Gilbert JR, Wilson DM, Best JA, Taylor DW, Lindsay EA, Singer J, Willms DG: Smoking cessation in primary care. A randomized controlled trial of nicotine-bearing chewing gum. Fam Pract 1989, 28:49-55.

46. Hughes JR, Gust SW, Keenan RM, Fenwick JW, Healey ML: Nicotine vs placebo gum in general medical practice. JAMA 1989 26 I: | 300-I305.

47. Killen JD, Fortmann SP, Newman B, Varady A: Evaluation of treatment approach combining nicotine gum with selfguided behavioral treatments for smoking relapse prevention. I Consult Clin Psychol 1990, 58:85-92.

48. Campbell IA, Prescott RJ, Tjeder-Burton SM: Smoking cessation in hospital patients given repeated advice plus nicotine or placebo chewing gum. Respir Med 199I, 85: I55-I57.

49. Segnan N, Ponti A, Battista RN, Senore C, Rosso S, Shapiro SH, Aimar $D$ : A randomized trial of smoking cessation interventions in general practice in Italy. Cancer Causes Control I991, 2:239-246.

50. Tonnesen P, Norregaard J, Simonsen K, Sawe U: A double-blind trial of a I6-hour transdermal nicotine patch in smoking cessation. $N$ Engl J Med I99I, 325:3।I-3I5.

51. McGovern PG, Lando HA: An assessment of nicotine gum as an adjunct to freedom from smoking cessation clinics. Addict Behav 1992, I7:137-147.

52. Nebot M, Cabezas C: Does nurse counseling or offer of nicotine gum improve the effectiveness of physician smoking-cessation advice? Fam Pract Res J 1992, I 2:263-270.

53. Pirie PL, McBride CM, Hellerstedt W, Jeffery RW, Hatsukami D, Allen $\mathrm{S}$, Lando $\mathrm{H}$ : Smoking cessation in women concerned about weight. Am J Public Health 1992, 82: I238-I 243.

54. Sutherland G, Stapleton IA, Russell MA, Jarvis MJ, Hajek P, Belcher M, Feyerabend C: Randomised controlled trial of nasal nicotine spray in smoking cessation. Lancet 1992, 340:324-329.

55. Zelman DC, Brandon TH, Jorenby DE, Baker TB: Measures of affect and nicotine dependence predict differential response to smoking cessation treatments. J Consult Clin Psychol 1992, 60:943-952.

56. Effectiveness of a nicotine patch in helping people stop smoking: results of a randomised trial in general practice. Imperial Cancer Research Fund General Practice Research Group. BM/ 1993, 306:1304-1308.

57. Sachs DP, Sawe U, Leischow SJ: Effectiveness of a 16-hour transdermal nicotine patch in a medical practice setting, without intensive group counseling. Arch Intern Med 1993, 153:1881-1890.

58. Tonnesen P, Norregaard J, Mikkelsen K, Jorgensen S, Nilsson F: A double-blind trial of a nicotine inhaler for smoking cessation. JAMA 1993, 269:1268-127|.

59. Hjalmarson A, Franzon M, Westin A, Wiklund O: Effect of nicotine nasal spray on smoking cessation. A randomized, placebocontrolled, double-blind study. Arch Intern Med 1994 I 54:2567-2572.

60. Hurt RD, Dale LC, Fredrickson PA, Caldwell CC Lee GA Offord KP, Lauger GG, Marusic Z, Neese LW, Lundberg TG: Nicotine patch therapy for smoking cessation combined with physician advice and nurse follow-up. One-year outcome and percentage of nicotine replacement. JAMA 1994, 27 I:595-600.

61. Niaura R, Goldstein MG, Abrams DB: Matching high- and lowdependence smokers to self-help treatment with or without nicotine replacement. Prev Med 1994, 23:70-77.

62. Dale LC, Hurt RD, Offord KP, Lawson GM, Croghan IT, Schroeder DR: High-dose nicotine patch therapy. Percentage of replacement and smoking cessation. JAMA 1995 274:1353-1358.

63. Fortmann SP, Killen JD: Nicotine gum and self-help behavioral treatment for smoking relapse prevention: results from a trial using population-based recruitment. J Consult Clin Psychol 1995, 63:460-468.

64. Herrera N, Franco R, Herrera L, Partidas A, Rolando R, Fagerstrom $K O$ : Nicotine gum, 2 and $4 \mathrm{mg}$, for nicotine dependence. A double-blind placebo-controlled trial within a behavior modification support program. Chest 1995, I08:447-45 I.

65. Kornitzer M, Boutsen M, Dramaix M, Thijs J, Gustavsson G: Combined use of nicotine patch and gum in smoking cessation: a placebo-controlled clinical trial. Prev Med 1995, 24:4I-47.

66. Schneider NG, Olmstead R, Mody FV, Doan K, Franzon M, Jarvik ME, Steinberg C: Efficacy of a nicotine nasal spray in smoking cessation: a placebo-controlled, double-blind trial. Addiction I995, 90: $167 \mid-1682$.

67. Stapleton JA, Russell MA, Feyerabend C, Wiseman SM, Gustavsson G, Sawe U, Wiseman D: Dose effects and predictors of outcome in a randomized trial of transdermal nicotine patches in general practice. Addiction 1995, 90:31-42.

68. Puska PKH, Vartiainen E, Urjanheimo E: Combined use of nicotine patch and gum compared with gum alone in smoking cessation: a clinical trial in North Karelia. Tabacco Control 1995, 4:23I-235.

69. Campbell IA, Prescott RJ, Tjeder-Burton SM: Transdermal nicotine plus support in patients attending hospital with smoking-related diseases: a placebo-controlled study. Respir Med 1996, 90:47-5।.

70. Cinciripini PM, Cinciripini LG, Wallfisch A, Haque W, Van Vunakis H: Behavior therapy and the transdermal nicotine patch: effects on cessation outcome, affect, and coping. J Consult Clin Psychol 1996, 64:3|4-323

7I. Hall SM, Munoz RF, Reus VI, Sees KL, Duncan C, Humfleet GL, Hartz $D T$ : Mood management and nicotine gum in smoking treatment: a therapeutic contact and placebo-controlled study. Consult Clin Psychol 1996, 64:1003-1009.

72. Paoletti P, Fornai E, Maggiorelli F, Puntoni R, Viegi G, Carrozzi L, Corlando A, Gustavsson G, Sawe U, Giuntini C: Importance of baseline cotinine plasma values in smoking cessation: results from a double-blind study with nicotine patch. Eur Respir J 1996, 9:643-65।.

73. Schneider NG, Olmstead R, Nilsson F, Mody FV, Franzon M, Doan K Efficacy of a nicotine inhaler in smoking cessation: a doubleblind, placebo-controlled trial. Addiction 1996, 91: |293-I306.

74. Leischow SJN, Franzo F, Hill M, Otte A, Merikle P, E P: Efficacy of the Nicotine Inhaler as an Adjunct to Smoking Cessation. American Journal of Health Behavior 1996, 20:364-371.

75. Blondal T, Franzon M, Westin A: A double-blind randomized trial of nicotine nasal spray as an aid in smoking cessation. Eur Respir J 1997, I 0: I585-I590.

76. Killen JD, Fortmann SP, Davis L, Varady A: Nicotine patch and selfhelp video for cigarette smoking cessation. J Consult Clin Psychol 1997, 65:663-672.

77. Hjalmarson A, Nilsson F, Sjostrom L, Wiklund O: The nicotine inhaler in smoking cessation. Arch Intern Med 1997, I57:172|-1728.

78. Richmond RL, Kehoe L, de Almeida Neto AC: Effectiveness of a 24-hour transdermal nicotine patch in conjunction with a cognitive behavioural programme: one year outcome. Addiction 1997, 92:27-31.

79. Daughton D, Susman J, Sitorius M, Belenky S, Millatmal T, Nowak R, Patil K, Rennard SI: Transdermal nicotine therapy and primary care. Importance of counseling, demographic, and participant selection factors on I-year quit rates. The Nebraska Primary Practice Smoking Cessation Trial Group. Arch Fam Med 1998, 7:425-430.

80. Perng RP, Hsieh WC, Chen YM, Lu CC, Chiang SJ: Randomized, double-blind, placebo-controlled study of transdermal nicotine patch for smoking cessation. I Formos Med Assoc 1998 , 97:547-55।

8I. Blondal T, Gudmundsson LJ, Olafsdottir I, Gustavsson G, Westin A Nicotine nasal spray with nicotine patch for smoking cessation: randomised trial with six year follow up. BMJ 1999 , 3 1 8:285-288. 
82. Jorenby DE, Leischow SJ, Nides MA, Rennard SI, Johnston JA, Hughes AR, Smith SS, Muramoto ML, Daughton DM, Doan K, et al.: A controlled trial of sustained-release bupropion, a nicotine patch, or both for smoking cessation. N Engl J Med I999, 340:685-69|.

83. Niaura R, Abrams DB, Shadel WG, Rohsenow DJ, Monti PM, Sirota $A D$ : Cue exposure treatment for smoking relapse prevention: a controlled clinical trial. Addiction 1999, 94:685-695.

84. Tonnesen P, Paoletti P, Gustavsson G, Russell MA, Saracci R, Gulsvik A, Rijcken B, Sawe U: Higher dosage nicotine patches increase one-year smoking cessation rates: results from the European CEASE trial. Collaborative European Anti-Smoking Evaluation. European Respiratory Society. Eur Respir J 1999 I 3:238-246

85. Bolliger CT, Zellweger JP, Danielsson $T$, van Biljon $X$, Robidou A, Westin A, Perruchoud AP, Sawe U: Smoking reduction with ora nicotine inhalers: double blind, randomised clinical trial of efficacy and safety. BM/ 2000, $321: 329-333$.

86. Bohadana A, Nilsson F, Rasmussen T, Martinet Y: Nicotine inhaler and nicotine patch as a combination therapy for smoking cessation: a randomized, double-blind, placebo-controlled trial. Arch Intern Med 2000, 160:3।28-3134.

87. Garvey AJ, Kinnunen T, Nordstrom BL, Utman CH, Doherty K, Rosner B, Vokonas PS: Effects of nicotine gum dose by level of nicotine dependence. Nicotine Tob Res 2000, 2:53-63.

88. Tonnesen P, Mikkelsen KL: Smoking cessation with four nicotine replacement regimes in a lung clinic. Eur Respir J 2000 16:717-722

89. Wallstrom M, Nilsson F, Hirsch JM: A randomized, double-blind, placebo-controlled clinical evaluation of a nicotine sublingual tablet in smoking cessation. Addiction 2000, 95: I |6I-I I7|

90. Wisborg K, Henriksen TB, Jespersen LB, Secher NJ: Nicotine patches for pregnant smokers: $A$ randomized controlled study. Obstet Gynecol 2000, 96:967-97।.

91. Glover ED, Glover PN, Franzon M, Sullivan CR, Cerullo CC, Howell RM, Keyes GG, Nilsson F, Hobbs GR: A comparison of a nicotine sublingual tablet and placebo for smoking cessation. Nicotine Tob Res 2002, 4:44I-450.

92. Hand S, Edwards S, Campbell IA, Cannings R: Controlled trial of three weeks nicotine replacement treatment in hospital patients also given advice and support. Thorax 2002 57:7|5-7|8.

93. Shiffman S, Dresler CM, Hajek P, Gilburt SJ, Targett DA, Strahs KR: Efficacy of a nicotine lozenge for smoking cessation. Arch Intern Med 2002, I 62: I 267-I276.

94. Glavas D, Rumboldt M, Rumboldt Z: Smoking cessation with nicotine replacement therapy among health care workers: randomized double-blind study. Croat Med J 2003, 44:219-224.

95. Molyneux A, Lewis S, Leivers U, Anderton A, Antoniak M, Brackenridge $A$, Nilsson F, McNeill A, West R, Moxham J, et al.: Clinical trial comparing nicotine replacement therapy (NRT) plus brief counselling, brief counselling alone, and minimal intervention on smoking cessation in hospital inpatients. Thorax 2003, 58:484-488.

96. Wennike P, Danielsson T, Landfeldt B, Westin A, Tonnesen P: Smoking reduction promotes smoking cessation: results from a double blind, randomized, placebo-controlled trial of nicotine gum with 2-year follow-up. Addiction 2003, 98: | 395-| 402

97. Swanson NA, Burroughs CC, Long MA, Lee RW: Controlled trial for smoking cessation in a Navy shipboard population using nicotine patch, sustained-release buproprion, or both. Mil Med 2003, I 68:830-834

98. Batra A, Klingler K, Landfeldt B, Friederich HM, Westin A, Danielsson $\mathrm{T}$ : Smoking reduction treatment with 4-mg nicotine gum: a double-blind, randomized, placebo-controlled study. Clin Pharmacol Ther 2005, 78:689-696.

99. Cooper TV, Klesges RC, Debon MW, Zbikowski SM, Johnson KC, Clemens LH: A placebo controlled randomized trial of the effects of phenylpropanolamine and nicotine gum on cessation rates and postcessation weight gain in women. Addict Behav 2005, 30:61-75.

100. Hall SM, Humfleet GL, Reus VI, Munoz RF, Hartz DT, Maude-Griffin $\mathrm{R}$ : Psychological intervention and antidepressant treatment in smoking cessation. Arch Gen Psychiatry 2002, 59:930-936.

101. Holt S, Timu-Parata C, Ryder-Lewis S, Weatherall M, Beasley R: Efficacy of bupropion in the indigenous Maori population in New Zealand. Thorax 2005, 60:120-123.
102. Hurt RD, Sachs DP, Glover ED, Offord KP, Johnston JA, Dale LC, Khayrallah MA, Schroeder DR, Glover PN, Sullivan CR, et al.: A comparison of sustained-release bupropion and placebo for smoking cessation. N Engl J Med I997, 337: I |95-I 202.

103. Simon JA, Duncan C, Carmody TP, Hudes ES: Bupropion for smoking cessation: a randomized trial. Arch Intern Med 2004, | 64: $1797-1803$

104. Tonnesen P, Tonstad S, Hjalmarson A, Lebargy F, Van Spiegel PI, Hider A, Sweet R, Townsend J: A multicentre, randomized, double-blind, placebo-controlled, I-year study of bupropion SR for smoking cessation. J Intern Med 2003, 254:184-192.

105. Tonstad S, Farsang C, Klaene G, Lewis K, Manolis A, Perruchoud AP, Silagy C, van Spiegel PI, Astbury C, Hider A, et al.: Bupropion SR for smoking cessation in smokers with cardiovascular disease: a multicentre, randomised study. Eur Heart J 2003, 24:946-955.

106. Zellweger JP, Boelcskei PL, Carrozzi L, Sepper R, Sweet R, Hider AZ: Bupropion SR vs placebo for smoking cessation in health care professionals. Am J Health Behav 2005, 29:240-249.

107. Klesges RC, Johnson KC, Somes G: Varenicline for smoking cessation: definite promise, but no panacea. JAMA 2006, 296:94-95.

108. Lando H: Data collection and questionnaire design: Smoking cessation in adults. In Measurement in the Analysis and Treatment of Smoking Behaviour Edited by: Grubowski J, CS CB. NIDA Research Monograph 48: U.S. Department of Health and Human Services, Public Health Service, Alcohol, Drug Abuse, and Mental Health Administration, National Institute on Drug Abuse; 1983:74-89.

109. Surgeon General: The Health Benefits of Smoking Cessation: A report of the Surgeon General. DHSS Publication No. (CDC) 90-8416: U.S. Department of Health and Human Services (DHSS), Public health Service (PHS), Centre for Disease Control (CDC), Centre for Chronic Disease Prevention and Health Promotion, Office on Smoking and Health; 1990.

110. Schulz KF, Chalmers I, Hayes RJ, Altman DG: Empirical evidence of bias. Dimensions of methodological quality associated with estimates of treatment effects in controlled trials. JAMA 1995, 273:408-4I2

I II. loannidis JP: Indirect comparisons: the mesh and mess of clinical trials. Lancet 2006, 368: |470-2.

I I2. Doll R, Peto R, Wheatley K, Gray R, Sutherland I: Mortality in relation to smoking: $\mathbf{4 0}$ years' observations on male British doctors. BM] 1994, 309:901-9|I.

I 13. Anthonisen NR, Connett JE, Kiley JP, Altose MD, Bailey WC, Buist AS, Conway WA Jr, Enright PL, Kanner RE, O'Hara P, et al.: Effects of smoking intervention and the use of an inhaled anticholinergic bronchodilator on the rate of decline of FEVI. The Lung Health Study. JAMA 1994, 272: |497-I505.

I 14. Wilson K, Gibson N, Willan A, Cook D: Effect of smoking cessation on mortality after myocardial infarction: meta-analysis of cohort studies. Arch Intern Med 2000, I 60:939-944.

II5. LaCroix AZ, Lang J, Scherr P, Wallace RB, Cornoni-Huntley J, Berkman L, Curb JD, Evans D, Hennekens $\mathrm{CH}$ : Smoking and mortality among older men and women in three communities. $N$ Engl J Med 1991, 324:1619-1625.

I 16. Ascher JA, Cole JO, Colin JN, Feighner JP, Ferris RM, Fibiger HC, Golden RN, Martin P, Potter WZ, Richelson E, et al.: Bupropion: a review of its mechanism of antidepressant activity. J Clin Psychiatry 1995, 56:395-401.

I17. Joseph AM, Norman SM, Ferry LH, Prochazka AV, Westman EC, Steele BG, Sherman SE, Cleveland M, Antonnucio DO, Hartman N, et al.: The safety of transdermal nicotine as an aid to smoking cessation in patients with cardiac disease. N Engl J Med I996, 335:1792-1798

\section{Pre-publication history}

The pre-publication history for this paper can be accessed here:

http://www.biomedcentral.com/1471-2458/6/300/pre pub 